\title{
General anesthetic management of Prader-Willi syndrome patient undergoing middle cerebral artery-superficial temporal artery anastomosis
}

\author{
Jin Young Lee, Kwang Rae Cho, Myoung Hun Kim, Kun Moo Lee, and Hyo Joong Kim \\ Department of Anesthesiology and Pain Medicine, Busan Paik Hospital, College of Medicine, Inje University, Busan, Korea
}

We experienced a case of a 17-year-old patient with genetically proven Prader-Willi syndrome who had left middle cerebral artery-left superficial temporal artery anastomosis surgery under general anesthesia with a non-depolarizing muscle relaxant. Prader-Willi syndrome is a genetic disorder associated with hypotonia. Using general anesthesia with a non-depolarizing muscle relaxant in these patients is difficult due to hypotonia. We, therefore, monitored the status of muscle relaxation with a train of four (TOF, TOF-Watch ${ }^{\circledR}$, Organon, Ireland) during general anesthesia. Unlike infants, adolescents can have the muscle relaxation monitor on their body surface.

Because lengthy, complex microsurgery was required for this vascular surgery, we had to use a muscle relaxant. However, when using a muscle relaxant, predicting the extent of the effect and the recovery time are difficult.

The TOF ratio was 0.85 in the preanesthetic state. Anesthetic induction was done using the total intravenous anesthesia of the target concentration infusion method. Propofol target concentration at $4 \mu \mathrm{g} / \mathrm{ml}$ was initially injected. Remifentanil was injected at $0.15 \mu \mathrm{g} / \mathrm{kg} / \mathrm{min}$. Five minutes after an atracurium $15 \mathrm{mg}$ injection, the TOF watch count was still at " 2 ". Therefore, an additional $15 \mathrm{mg}$ of atracurium was injected for intubation. When the TOF watch count was over " 4 ", during the operation, an additional $5 \mathrm{mg}$ of atracurium was injected. For the entire surgery, $65 \mathrm{mg}$ of atracurium was used.

The operation lasted for 2 hours and 50 minutes. After the operation, when the TOF ratio was 0.85 , glycopyrrolate $0.4 \mathrm{mg}$ and pyridostigmine $15 \mathrm{mg}$ were injected. The endotracheal tube was removed once the patient regained spontaneous respiration and was fully awake. After extubation, the patient was arousable using painful stimulation. In the post anesthetic care unit, the patient had no respiratory problem and his TOF ratio was 0.85 . The patient was then transferred to the general ward.

Prader-Willi syndrome is a genetic disorder classically characterized by hypotonia, hypomentia, and hypogonadism that is associated with obesity [1]. The cause of hypotonia, muscle weakness, and motor problems in Prader-Willi patients are not clear. The contributing factors may be the abnormal body composition found in Prader-Willi patients, an increase in fat mass and a decrease in muscle mass, and, possibly, some degree of neuromuscular abnormality [2]. It has been reported that the motor problems are still present in childhood and adulthood, which causes the decrease in physical activity in Prader-Willi patients [3]. Thus, the hypotonia makes the choice of muscle relaxant difficult. Anesthetic management using atracurium and rocuronium have been reported [4,5]. However, few reports are available on using the intraoperative monitoring of the neuromuscular blockade for Prader-Willi patients.

In this case, though the patient had hypotonia, anesthesia was successfully used with a dose adjusted muscle relaxant using the TOF watch.

Corresponding author: Kwang Rae Cho, M.D., Department of Anesthesiology and Pain Medicine, Busan Paik Hospital, College of Medicine, Inje University, Gaegeum 2-dong, Busanjin-gu, Busan 614-735, Korea. Tel: 82-51-890-6520, Fax: 82-51-898-4216, E-mail: maeteo@hanmir.com (c) This is an open-access article distributed under the terms of the Creative Commons Attribution Non-Commercial License (http:// creativecommons.org/licenses/by-nc/3.0/), which permits unrestricted non-commercial use, distribution, and reproduction in any medium, provided the original work is properly cited. 


\section{References}

1. Legrand R, Tobias JD. Anesthesia and Prader-Willi syndrome: preliminary experience with Regional anesthesia. Paediatr Anaesth 2006; 16: 712-22.

2. Lewis CL. Prader-Willi syndrome: a review for pediatric physical therapists. Pediatr Phys Ther 2000; 12: 87-95.

3. Butler MG, Theodoro MF, Bittel DC, Donnelly JE. Energy expen- diture and physical activity in Prader-Willi syndrome: comparison with obese subjects. Am J Med Genet A 2007; 143: 449-59.

4. Dearlove OR, Dobson A, Super M. Anesthesia and Prader-Willi syndrome. Paediatr Anaesth 1998; 8: 267-71.

5. Shrarma AD, Erb T, Schulman SR, Sreeram G, Slaughter TF. Anaesthetic considerations for a child with combined Prader-Willi syndrome and mitochondrial myopathy. Paediatr Anaesth 2001; 11 : 488-90. 